\title{
MATЕMАТИКА
}

УДК $517.55+519.85+519.111 .1$

DOI $10.18413 / 2687-0959-2020-52-1-5-10$

\section{ВЫЧИСЛЕНИЕ ПОСЛЕДОВАТЕЛЬНОСТИ ГЛАВНЫХ МИНОРОВ ТЕПЛИЦЕВОЙ ЛЕНТОЧНОЙ МАТРИЦЫ}

\author{
М. С. Апанович ${ }^{1}$, А. П. Ляпин ${ }^{2}$, К. В. Шадрин ${ }^{1}$ \\ Статъл представлена членом редакционной коллегии С. М. Ситником \\ ${ }^{1}$ Красноярский государственный медицинский университет имени профессора В. Ф. Войно-Ясенецкого \\ г. Красноярск, 660022, Россия \\ 2 Сибирский федеральный университет \\ г. Красноярск, 660041, Россия \\ E-mail: marina.apanovich@list.ru, aplyapin@sfu-kras.ru, kvsh_buffon@mail.ru
}

\begin{abstract}
Аннотация. В работе рассматривается алгоритм, который позволяет вычислить последовательность главных миноров теплицевой ленточной матрицы, ассоциированной с задачей Коши для двумерного полиномиального разностного оператора с постоянными коэффициентами, с верхней (нижней) шириной ленты равной единице, что позволит определить невырожденность такой матрицы и, следовательно, сделать выводы о разрешимости задачи Коши.
\end{abstract}

Ключевые слова: полиномиальный разностный оператор, задача Коши, разрешимость, ленточная матрица, теплицева матрица, главные миноры.

Благодарности: работа первого автора выполнена при финансовой поддержке РФФИ в рамках научного проекта №18-31-00232.

Для цитирования: Апанович М. С., Ляпин А. П., Шадрин К. В. 2020. Вычисление последовательности главных миноров теплицевой ленточной матрицы. Прикладная математика \& Физика. 52(1): 5-10.

DOI 10.18413/2687-0959-2020-52-1-5-10.

\section{CALCULATING THE SEQUENCE OF MAIN MINORS OF THE TOEPLITZ BAND MATRIX}

\author{
M. S. Apanovich ${ }^{1}$, A. P. Lyapin ${ }^{2}$, K. V. Shadrin ${ }^{1}$ \\ Article submitted by a member of the editorial board S. M. Sitnik \\ ${ }^{1}$ Krasnoyarsk State Medical University named after Prof. V. F. Voino-Yasenetsky, \\ Krasnoyarsk, 660022, Russia \\ ${ }^{2}$ Siberian Federal University \\ Krasnoyarsk, 660041, Russia \\ E-mail: marina.apanovich@list.ru, aplyapin@sfu-kras.ru, kvsh_buffon@mail.ru
}

Received March 18, 2020

\begin{abstract}
In this paper, we consider an algorithm that allows you to calculate the sequence of the main minors of the Toeplitz band matrix associated with the Cauchy problem for a two-dimensional polynomial difference operator with constant coefficients, with an upper (lower) band width equal to one, which allows us to determine the non-degeneracy of such a matrix and, therefore, draw conclusions on the solvability of the Cauchy problem

Key words: polynomial difference operator, Cauchy problem, solvability, band matrix, toeplitz matrix, main minor.

Acknowledgements: The work $\mathrm{f}$ the first author is supported by the Russian Federal Property Fund in the framework of scientific project textnumero 18-31-00232.

For citation: Apanovich M. S., Lyapin A. P., Shadrin K. V. 2020. Calculating the sequence of main minors of the toeplitz band matrix. Applied Mathematics \& Physics. 52(1): 5-10 (in Russian).

DOI 10.18413/2687-0959-2020-52-1-5-10.
\end{abstract}


1. Введение. Линейные разностные уравнения (уравнения в конечных разностях или рекуррентные уравнения) возникают в различных областях математики. Например, разностные уравнения часто используются в моделях динамики при моделировании процессов с дискретным временем ([3], [4]), а также для приближенного решения дифференциальных уравнений ([10]), в комбинаторном анализе в сочетании с методом производящих функций дают мощный аппарат исследования перечислительных задач (см., например, [13], [14]), следовательно, поиск решений таких уравнений является одной из математических задач, имеющей многочисленные приложения в различных областях науки и техники [8], [11], [12].

В работе [15] рассмотрен вариант задачи Коши для многомерного разностного уравнения с постоянными коэффициентами, возникающий с задачей о числе путей на целочисленной решетке в перечислительном комбинаторном анализе. Получена формула, выражающая производящую функцию решения задачи Коши через производящие функции данных Коши, и найдено решение задачи Коши через ее фундаментальное решение и данные Коши.

В работе [9] в связи с исследованием разрешимости задачи Коши для двумерного разностностного оператора вводится понятие ассоциированной матрицы, которая представлеят собой теплицевую ленточную матрицу бесконечного порядка, и ее невырожденность является необходимым и достаточным условием разрешимости задачи Коши, а также было доказано рекурретное соотношение для последовательности главных миноров теплицевой ленточной матрицы.

Данная работа посвящена описанию алгоритма для вычисления последовательности главных миноров теплицевой ленточной матрицы с верхней (нижней) шириной ленты равной единице, что позволит определить невырожденность такой матрицы и, следовательно, сделать выводы о разрешимости задачи Коши. Кроме того, приводится пример, иллюстрирующий его работу.

\section{2. Постановка задачи и известные результаты.}

Определение 1. Матрица $A=\left\|a_{i j}\right\|$ называется ленточной, если все ненулевые элементы заключены внутри ленты, образованной между диагоналями, параллельными главной. Если для матрицы $A$ справедливо: $a_{i j}=0$ при $i>j+l$ и $a_{i j}=0$ при $j>i+k$, то $l$ называется нижней шириной ленты, $k$ - верхней шириной ленты. Величина $s=l+k+1$ называется шириной ленты матрицы $A$.

Оперделение 2. Матрица $B$ называется теплицевой (см., например, [5]), если на всех диагоналях матрицы $\left\|b_{i j}\right\|$, параллельных главной диагонали и на самой главной диагонали, элементы матрицы одинаковы: $b_{i j}=b_{j-i}$.

Обозначим через $\mathbb{Z}^{2}$ двумерную целочисленную решетку и $\mathbb{Z}_{\geqslant}^{2}-$ подмножество этой решетки, состоящее из точек с целыми неотрицательными координатами. Пусть $\delta_{1}$ - оператор сдвига по переменной $x_{1}$, т.е. $\delta_{1} f\left(x_{1}, x_{2}\right)=f\left(x_{1}+1, x_{2}\right)$, а $\delta_{2}-$ оператор сдвига по переменной $x_{2}$, т.е. $\delta_{2} f\left(x_{1}, x_{2}\right)=f\left(x_{1}, x_{2}+1\right)$.

Рассмотрим полиномиальный разностный оператор вида

$$
P\left(\delta_{1}, \delta_{2}\right)=\sum_{\alpha_{1}+\alpha_{2} \leqslant m} c_{\alpha_{1}, \alpha_{2}} \delta_{1}^{\alpha_{1}} \delta_{2}^{\alpha_{2}},
$$

где $c_{\alpha_{1}, \alpha_{2}}$ - постоянные коэффициенты, $m$ - порядок оператора $P\left(\delta_{1}, \delta_{2}\right)$.

Будем рассматривать разностные уравнения вида

$$
P\left(\delta_{1}, \delta_{2}\right) f\left(x_{1}, x_{2}\right)=0, \quad\left(x_{1}, x_{2}\right) \in \mathbb{Z}_{\geqslant}^{2}
$$

где $f\left(x_{1}, x_{2}\right)$ - неизвестная функция.

Для точек $x=\left(x_{1}, x_{2}\right)$ и $y=\left(y_{1}, y_{2}\right)$ решетки $\mathbb{Z}^{2}$ запись $x \ngtr y$ означает, что найдется $i_{0} \in\{1,2\}$ такое, что $x_{i_{0}}<y_{i_{0}}$.

Зафиксируем $\beta=(l, k)$ такое, что $l+k+1=m$ и $c_{l, k} \neq 0$ и сформулируем задачу Коши:

найти решение разностного уравнения (1), удовлетворяющее условию

$$
f\left(x_{1}, x_{2}\right)=\varphi\left(x_{1}, x_{2}\right), \quad\left(x_{1}, x_{2}\right) \ngtr(l, k) .
$$

Переобозначим коэффициенты $c_{\alpha_{1}, \alpha_{2}}$ однородной составляющей $\sum_{\alpha_{1}+\alpha_{2}=m} c_{\alpha_{1}, \alpha_{2}} \delta_{1}^{\alpha_{1}} \delta_{2}^{\alpha_{2}}$ старшей степени многочлена $P\left(\delta_{1}, \delta_{2}\right)$ :

$$
\begin{gathered}
t_{-l}=c_{0, m}, t_{-l+1}=c_{1, m-1}, \ldots, t_{-1}=c_{-l+1, k+1}, t_{0}=c_{l, k}, \\
t_{1}=c_{l+1, k-1}, \ldots, t_{k-1}=c_{m-1,1}, t_{k}=c_{m, 0} .
\end{gathered}
$$

Ассоциированной с задачей (1) - (2) матрицей будем называть матрицу бесконечного порядка вида: 


$$
\mathrm{T}_{\infty}=\left\|\begin{array}{ccccccc}
t_{0} & t_{1} & t_{2} & \cdots & t_{k} & 0 & \cdots \\
t_{-1} & t_{0} & t_{1} & \ddots & \vdots & t_{k} & \ddots \\
t_{-2} & t_{-1} & \ddots & \ddots & t_{2} & \vdots & \ddots \\
\vdots & \ddots & \ddots & \ddots & t_{1} & t_{2} & \vdots \\
t_{-l} & \cdots & t_{-2} & t_{-1} & t_{0} & t_{1} & \ddots \\
0 & t_{-l} & \cdots & t_{-2} & t_{-1} & t_{0} & \ddots \\
\vdots & \ddots & \ddots & \cdots & \ddots & \ddots & \ddots
\end{array}\right\| .
$$

Далее обозначим $D_{p}$ - главный минор матрицы $\mathrm{T}_{\infty}$ порядка $p$. Например, для разностного оператора

$$
P\left(\delta_{1}, \delta_{2}\right)=t_{2} \delta_{1}^{3}+t_{1} \delta_{1} \delta_{2}^{2}+t_{0} \delta_{1}^{2} \delta_{2}+t_{-1} \delta_{2}^{3}+\sum_{\alpha_{1}+\alpha_{2} \leqslant 2} c_{\alpha_{1}, \alpha_{2}} \delta_{1}^{\alpha_{1}} \delta_{2}^{\alpha_{2}}
$$

ассоциированная матрица имеет вид

$$
T_{\infty}=\left\|\begin{array}{ccccc}
t_{0} & t_{1} & t_{2} & 0 & \cdots \\
t_{-1} & t_{0} & t_{1} & t_{2} & \ddots \\
0 & t_{-1} & t_{0} & t_{1} & \ddots \\
0 & 0 & t_{-1} & t_{0} & \ddots \\
\vdots & \ddots & \ddots & \ddots & \ddots
\end{array}\right\|,
$$

а главные миноры

$$
D_{1}=t_{0}, D_{2}=\left|\begin{array}{cc}
t_{0} & t_{1} \\
t_{-1} & t_{0}
\end{array}\right|, D_{3}=\left|\begin{array}{ccc}
t_{0} & t_{1} & t_{2} \\
t_{-1} & t_{0} & t_{1} \\
0 & t_{-1} & t_{0}
\end{array}\right|, D_{4}=\left|\begin{array}{cccc}
t_{0} & t_{1} & t_{2} & 0 \\
t_{-1} & t_{0} & t_{1} & t_{2} \\
0 & t_{-1} & t_{0} & t_{1} \\
0 & 0 & t_{-1} & t_{0}
\end{array}\right|, \ldots
$$

Приведем необходимое и достаточное условие разрешимости задачи Коши (1) - (2) из работы [9]: задача (1) - (2) имеет единственное решение тогда и только тогда, когда ассоциированная матрица $\mathrm{T}_{\infty}$ невырожденная, т.е. для любого $p$ главные миноры $D_{p} \neq 0$. Кроме того, в работе [9] было доказано рекурретное соотношение для последовательности главных миноров теплицевой ленточной матрицы, а именно: последовательность $D_{p}$ главных миноров теплицевой ленточной матрицы $\mathrm{T}_{\infty} \mathrm{c}$ верхней шириной ленты $k=1$ (нижней шириной ленты $l=1$ ) удовлетворяет рекуррентному соотношению порядка $l+1(k+1)$

$$
\begin{gathered}
D_{p}=t_{0} D_{p-1}+\sum_{v=1}^{l}(-1)^{v} t_{1}^{v} t_{-v} D_{p-1-v}, p=3,4, \ldots, l=1,2, \ldots \\
\left(D_{p}=t_{0} D_{p-1}+\sum_{v=1}^{k}(-1)^{v} t_{-1}^{v} t_{v} D_{p-1-v}, p=3,4, \ldots, k=1,2, \ldots\right) .
\end{gathered}
$$

3. Описание алгоритма. Для построения последовательности главных миноров теплицевой ленточной матрицы нам надо знать:

1) точку $\beta$, определяющую количество наддиагоналей и поддиагоналей теплицевой ленточной матрицы, т.е. ширину ленты. Поскольку рекуррентное соотношение для последовательности главных миноров в работе [9] было доказано для теплицевой ленточной матрицы, имеющей одну наддиагональ или одну поддиагональ, то одна из координат точки $\beta$ должна быть равна единице.

2) коэффициенты двумерного разностного оператора. Коэффициенты двумерного разностного оператора задаются квадратной матрицей $C$, имеющей нижнетреугольный вид.

3) количество элементов последовательности главных миноров, оно может быть много больше,чем размерость теплицевой ленточной матрицы, построенной по коэффициентам разностного опертора.

Итак, входные данные конечны и имееют вид:

1) точка $\beta=(l, k)$, где $l=1$ или $k=1$;

2 ) нижнетреугольная матрица $C=\left(c_{\alpha_{1}, \alpha_{2}}\right), \alpha_{1}=0, \ldots, m, \alpha_{2}=0, \ldots, m$, размера $(m+1) \times$ $(m+1)$ из коэффициентов $c_{\alpha_{1}, \alpha_{2}}$ двумерного разностного оператора; 
3) количество элементов последовательности главных миноров.

Отметим, что координаты точек в декартовой системе координат отличаются от координат точек в «матричной» системе координат и потребуется их перевод из одной системы координат в другую. Далее необходимо будет проверить, что одна из координат точки $\beta$ равна единице.

Соответствующий алгоритм размещен по ссылке https://github.com/lyapinap/ALS2020.

4. Пример работы алгоритма. Алгоритм был реализован в среде Matlab 201432 bit. Вычисления производились на машине Intel(R) Core(TM) i5-3330S CPU 2.70 GHz, 32bit, O3У 4.00 Гб под управлением Windows 7 Kорпоративная SP1. Время счета для приведенного примера составило менее 1 секунды.

Рассмотрим полиномиальный разностный оператор

$$
\begin{aligned}
& P\left(\delta_{1}, \delta_{2}\right)=c_{03} \delta_{2}^{3}+c_{12} \delta_{1} \delta_{2}^{2}+c_{21} \delta_{1}^{2} \delta_{2}+c_{03} \delta_{1}^{3}+ \\
& +c_{02} \delta_{2}^{2}+c_{11} \delta_{1} \delta_{2}+c_{20} \delta_{1}^{2}+c_{01} \delta_{2}+c_{10} \delta_{1}+c_{00}
\end{aligned}
$$

Фиксируем $\beta=(1,2)$, тогда теплицевая ленточная матрица будет иметь одну поддиагональ $(l=1)$ и две наддиагонали $(k=2)$, а ширина ленты будет равна $l+k+1=4$.

Зададим матрицу коэффициентов полиномиального разностного оператора $P\left(\delta_{1}, \delta_{2}\right)$

$$
C=\left(\begin{array}{cccc}
c_{03} & 0 & 0 & 0 \\
c_{02} & c_{12} & 0 & 0 \\
c_{01} & c_{11} & c_{21} & 0 \\
c_{00} & c_{10} & c_{20} & c_{30}
\end{array}\right)=\left(\begin{array}{cccc}
1 & 0 & 0 & 0 \\
2 & 10 & 0 & 0 \\
3 & 9 & 8 & 0 \\
4 & 5 & 6 & 7
\end{array}\right)
$$

Поставим задачу найти первые шесть элементов последовательности главных миноров.

Вектор диагонали матрицы коэффициентов $C: a=\left(\begin{array}{llll}1 & 10 & 8 & 7\end{array}\right)^{T}$.

Получение общей теплицевой матрицы.

$$
\begin{gathered}
\text { colomn }_{c}=\left(\begin{array}{llll}
10 & 1 & 0 & 0
\end{array}\right)^{T} \\
\text { row }_{c}=\left(\begin{array}{llll}
10 & 8 & 7 & 0
\end{array}\right)^{T} \\
T_{4}=\left(\begin{array}{cccc}
10 & 8 & 7 & 0 \\
1 & 10 & 8 & 7 \\
0 & 1 & 10 & 8 \\
0 & 0 & 1 & 10
\end{array}\right)
\end{gathered}
$$

Рузультатом работы алгоритма является вектор с главными минорами:

$$
\left(\begin{array}{llllll}
10 & 92 & 847 & 7804 & 71908 & 662577
\end{array}\right) .
$$

Итоговый вектор с шестью первыми элементами последовательности главных миноров не содержит нулевых элементов, кроме того, каждый следующий элемент последовательности больше предыдущего, таким образом, можно сделать вывод, что задача Коши для рассматриваемого двумерного разностного оператора разрешима. Для вычисления производящей функции решения задачи Коши для двумерного разностного оператора с постоянными коэффициентами можно воспользоваться программой [7], принцип работы которой описан в работе [6], а для вычисления решения задачи Коши для двумерного разностного оператора с постоянными коэффициентами можно использовать программу [1]. Для вычисления последовательности главных миноров можно использовать программу [2], принцип работы которой и описан в данной статье.

5. Заключение. В работе рассмотрен алгоритм вычисления последовательности главных миноров теплицевой ленточной матрицы, ассоциированной с задачей Коши, для двумерного разностного оператора и иллюстрирующий его работу пример.

\section{Список литературы}

1. Апанович M. С., Шадрин К. В. 2019. CauchyTriangle. Свидетельство о государственной регистрации программы для ЭВМ №2019663199.

2. Апанович M. С., Шадрин K. В. 2020. ToeplitzBanDet. Свидетельство о государственной регистрации программы для ЭВМ №2020612776.

3. Даджион Д., Мерсеро Р. 1988. Цифровая обработка многомерных сигналов. Пер. с англ. М., Мир, 487. (Dudgeon D. E., Mersereau R. M. 1983. Multidimensional digital signal processing. Englewood Cliffs, NJ : Prentice-Hall, 400.). 
4. Изерман Р. 1984. Цифровые системы управления. М., Мир, 541.

5. Иохвидов И. С. 1974. Ганкелевы и теплицевы матрицы. М., Наука, 264.

6. Kytmanov A. A., Lyapin A. P., Sadykov T. M. 2017. Evaluating the rational generating function for the solution of the Cauchy problem for a two-dimensional difference equation with constant coefficients. Programming and Computer Software, 43(2): 105-111.

7. Кытманов А. А., Ляпин А. П., Садыков Т. М. 2018. Программа вычисления рациональной производящей функции решения задачи Коши двумерного разностного уравнения с постоянными коэффициентами. Свидетельство о государственной регистрации программы для ЭВМ №2018619622.

8. Рогозина М. С. 2012. Устойчивость многослойных разностных схем и амебы алгебраических гиперповерхностей. Журнал СФУ. Серия Математика и физика, 5(2): 256-263.

9. Рогозина М. С. 2014. О разрешимости задачи Коши для полиномиального разностного оператора. Вестник Новосибирского государственного университета, 14 (3): 83-94.

10. Рябенький В. С. 2000. Введение в вычислительную математику: учеб. пособие, изд. 2-е, исправл. М., ФИЗМАТЛИТ, 296 с.

11. Рябенький В. С., Филиппов А. Ф. 1956. Об устойчивости разностных уравнений. М., Гос. изд. технико-теоретической литературы, $171 \mathrm{c}$.

12. Самарский А.А. 1971. Введение в теорию разностных схем. М., Наука, 552.

13. Стенли Р. 1990. Перечислительная комбинаторика: Пер. с англ. М., Мир, 440. (Stanley R. 1997. Enumerative combinatorics. Cambridge University Press, 326).

14. Стенли Р. 2005. Перечислительная комбинаторика. Деревья, производящие функции и симметрические функции: Пер. с англ. М., Мир, 767. ( Stanley R. 1997. Enumerative combinatorics. Trees and the Composition of Generating Functions. Cambridge University Press, 600).

15. Lyapin A. P., Chandragiri S. 2020. The Cauchy Problem for Multidimensional Difference Equations in Lattice Cones. Journal of Siberian Federal University. Mathematics \& Physics, 13(2): 187-196.

\section{References}

1. Apanovich M.S., Shadrin K.V. 2019. CauchyTriangle. Svidetel'stvo o gosudarstvennoj registracii programmy dlja JeVM №2019663199.

2. Apanovich M.S., Shadrin K.V. 2020. ToeplitzBanDet. Svidetel'stvo o gosudarstvennoj registracii programmy dlja JeVM №2020612776.

3. Dadzhion D., Mersero O. 1988. Cifrovaja obrabotka mnogomernyh signalov [Multidimensional digital signal processing]. Moscow, Mir, 487. (Dudgeon D.E., Mersereau R.M. 1983. Multidimensional digital signal processing. Englewood Cliffs, NJ : Prentice-Hall, 400.).

4. Izerman R. 1984. Cifrovye sistemy upravlenija. Moscow, Mir, 541.

5. Iohvidov I. S. 1974. Gankelevy i teplicevy matricy. Moscow, Nauka, 264.

6. Kytmanov A. A., Lyapin A. P., Sadykov T. M. 2017. Evaluating the rational generating function for the solution of the Cauchy problem for a two-dimensional difference equation with constant coefficients. Programming and Computer Software, 43(2): 105-111.

7. Kytmanov A. A., Ljapin A. P., Sadykov T. M. 2018. Programma vychislenija racional'noj proizvodjashhej funkcii reshenija zadachi Koshi dvumernogo raznostnogo uravnenija s postojannymi kojefficientami. Svidetel’stvo o gosudarstvennoj registracii programmy dlja JeVM №2018619622.

8. Rogozina M. S. 2012. Ustojchivost' mnogoslojnyh raznostnyh shem i ameby algebraicheskih giperpoverhnostej. Zhurnal SFU. Serija Matematika i fizika, 5(2): 256-263.

9. Rogozina M. S. 2014. O razreshimosti zadachi Koshi dlja polinomial'nogo raznostnogo operatora. Vestnik Novosibirskogo gosudarstvennogo universiteta, 14 (3): 83-94.

10. Rjaben'kij V. S. 2000. Vvedenie v vychislitel'nuju matematiku: ucheb. posobie, izd. 2-e, ispravl. Moscow, FIZMATLIT, 296 p. 
11. Rjaben'kij V.S., Filippov A.F. 1956. Ob ustojchivosti raznostnyh uravnenij. M., Gos. izd. tehnikoteoreticheskoj literatury, 171.

12. Samarskij A.A. 1971. Vvedenie v teoriju raznostnyh shem, M., Nauka, 552.

13. Stenli R. 1990. Perechislitel'naja kombinatorika [Enumerative combinatorics]. Moscow, Mir, 440. (Stanley R. 1997. Enumerative combinatorics. Cambridge University Press, 326).

14. Stenli R. 2005. Perechislitel'naja kombinatorika. Derev'ja, proizvodjashhie funkcii i simmetricheskie funkcii [Enumerative combinatorics. Trees and the Composition of Generating Functions]. Moscow, Mir, 767. ( Stanley R. 1997. Enumerative combinatorics. Trees and the Composition of Generating Functions. Cambridge University Press, 600).

15. Lyapin A. P., Chandragiri S. 2020. The Cauchy Problem for Multidimensional Difference Equations in Lattice Cones. Journal of Siberian Federal University. Mathematics \& Physics, 13(2): 187-196.

Получена 18.03.2020

Апанович Марина Степановна - кандидат физико-математических наук, доцент кафедры медицинской кибернетики и информатики Красноярского государственного медицинского университета имени профессора В. Ф. Войно-Ясенецкого

ул. Партизана Железняка, 1, г. Красноярск, Россия, 660022

E-mail: marina.apanovich@list.ru

Ляпин Александр Петрович - кандидат физико-математических наук, доцент базовой кафедры вычислительных и информационных технологий Сибирского федерального университета

пр. Свободный, 79, г. Красноярск, Россия, 660041

E-mail: aplyapin@sfu-kras.ru

Шадрина Константин Викторович - старший преподаватель кафедры медицинской кибернетики и информатики Красноярского государственного медицинского университета имени профессора В. Ф. Войно-Ясенецкого

ул. Партизана Железняка, 1, г. Красноярск, Россия, 660022

E-mail: kvsh_buffon@mail.ru 